\title{
Correction to: A Semi-passive Planar Manipulandum for Upper-Extremity Rehabilitation
}

\author{
Chin-Kang Chang, ${ }^{1}$ Edward P. Washabaugh, ${ }^{1,2}$ Andrew Gwozdziowski, ${ }^{1}$ C. David Remy $, 3,4$ and \\ Chandramouli Krishnan (10 ${ }^{1,2,4}$ \\ ${ }^{1}$ Neuromuscular and Rehabilitation Robotics Laboratory (NeuRRo Lab), Department of Physical Medicine and Rehabilitation, \\ University of Michigan, Ann Arbor, MI, USA; ${ }^{2}$ Department of Biomedical Engineering, University of Michigan, Ann Arbor, \\ MI, USA; ${ }^{3}$ RAM Lab, Department of Mechanical Engineering, University of Michigan, Ann Arbor, MI, USA; and ${ }^{4}$ Michigan \\ Robotics, College of Engineering, University of Michigan, Ann Arbor, MI, USA
}

\section{Correction to:}

Annals of Biomedical Engineering,

Vol. 46, No. 7, July 2018, pp. 1047-1065

https://doi.org/10.1007/s10439-018-2020-z

Authors would like to correct their acknowledgments. Correct acknowledgments appear here.

\section{ACKNOWLEDGMENTS}

Research reported in this publication was supported by (1) Disability and Rehabilitation Engineering
(DARE) Program of the National Science Foundation (Award\# 1804053), (2) National Institute of Biomedical Imaging and Bioengineering (NIBIB) of the National Institutes of Health (Grant\# R01 EB019834), (3) National Science Foundation Graduate Research Fellowship Program under Grant No. DGE \#1256260, and (4) the University of Michigan Office of Research (UMOR) MCubed 2.0 program. The content is solely the responsibility of the authors and does not necessarily represent the official views of the funding agencies. A special thanks to Shannon Leon and Justin Lee for the work they did in building and programming the robot.

Address correspondence to Chandramouli Krishnan, Neuromuscular and Rehabilitation Robotics Laboratory (NeuRRo Lab), Department of Physical Medicine and Rehabilitation, University of Michigan, Ann Arbor, MI, USA. Electronic mail: mouli@umich.edu 1007/s10439-018-2020-z. 